\title{
Antimicrobial resistance and novel mutations detected in the gyrA and parC genes of Pseudomonas aeruginosa strains isolated from companion dogs
}

Youjin Park', Jaeyoung Oh', Sowon Park', Samuth Sum¹, Wonkeun Song ${ }^{2}$, Jongchan Chae ${ }^{3}$ and Heemyung Park ${ }^{1 *}$

\begin{abstract}
Background: Fluoroquinolone agents, such as enrofloxacin and marbofloxacin, are commonly used for pseudomonal infection in veterinary medicine. However, the rate of resistance to fluoroquinolones is rapidly increasing, according to multiple studies in various countries. Point mutations in the quinolone resistancedetermining region (QRDR) are closely related to the increased fluoroquinolone resistance of Pseudomonas aeruginosa. The aim of this study was to investigate current antimicrobial susceptibility and fluoroquinolone resistance in $P$. aeruginosa strains isolated from dogs. The presence of point mutations in the QRDR was confirmed by gyrA and parC polymerase chain reaction and nucleotide sequencing analysis.

Results: A total of 84 nonduplicated P. aeruginosa strains were obtained from 228 healthy dogs (healthy group) and 260 dogs with clinical signs (infected group). Among these isolates, 38 strains from the healthy group were detected in several sample types, whereas 46 strains from the infected group were obtained mostly from dogs' ears with otitis externa $(41 / 260,15.8 \%)$. All strains were resistant to nalidixic acid, while some were also resistant to enrofloxacin (23/84, 27.4\%), marbofloxacin (17/84, 20.2\%), levofloxacin (12/84, 14.3\%), or ciprofloxacin (11/84, 13.1\%). Enrofloxacin resistance was significantly higher in strains from the infected group than in those from the healthy group ( $p<0.05$ ). Among the 23 fluoroquinolone-resistant strains, 8 and 4 different mutations were detected in the gyrA and parC genes, respectively. Mutations in gyrA were significantly common in the infected group $(p<0.05)$. Hotspots for the gyrA and parC mutations were Thr83 (34.8\%, 8/23) and Pro116 (91.3\%, 21/23), respectively. Double and triple mutations were also found in 5 of the strains.
\end{abstract}

Conclusion: Novel mutations in the gyrA and parC genes were first found in $P$. aeruginosa isolated from companion dogs in South Korea. These findings suggest that it is important to encourage prudent use of fluoroquinolone antibiotics in canine pseudomonal infection treatment.

Keywords: Companion dogs, P. aeruginosa, Novel mutations, gyrA, parC

\footnotetext{
* Correspondence: parkhee@konkuk.ac.kr

'Department of Veterinary Internal Medicine, Konkuk University College of Veterinary Medicine, Seoul, South Korea

Full list of author information is available at the end of the article
}

(c) The Author(s). 2020 Open Access This article is licensed under a Creative Commons Attribution 4.0 International License, which permits use, sharing, adaptation, distribution and reproduction in any medium or format, as long as you give appropriate credit to the original author(s) and the source, provide a link to the Creative Commons licence, and indicate if changes were made. The images or other third party material in this article are included in the article's Creative Commons licence, unless indicated otherwise in a credit line to the material. If material is not included in the article's Creative Commons licence and your intended use is not permitted by statutory regulation or exceeds the permitted use, you will need to obtain permission directly from the copyright holder. To view a copy of this licence, visit http://creativecommons.org/licenses/by/4.0/. The Creative Commons Public Domain Dedication waiver (http://creativecommons.org/publicdomain/zero/1.0/) applies to the data made available in this article, unless otherwise stated in a credit line to the data. 


\section{Background}

Pseudomonas aeruginosa is a gram-negative opportunistic bacterium that infects usually the skin and the urinary and respiratory tracts. In veterinary medicine, fluoroquinolones, such as enrofloxacin and marbofloxacin, are commonly prescribed when a pseudomonal infection is suspected, most commonly for otitis externa/ media or urinary tract infections. However, caution should be exerted in the use of antimicrobials to treat pseudomonal infections because of the increasing emergence of antimicrobial-resistant bacteria. The inappropriate use of antimicrobials, increasing prevalence of chronic illness, and lack of environmental barriers between animals and humans has not only contributed to the selection of antimicrobial-resistant bacteria but also imposed a variety of selective pressures that bacteria must face and contend with [1-3].

Quinolones are broad-spectrum antimicrobials that inhibit bacterial DNA gyrase and type IV topoisomerase. In human medicine, the trend of $P$. aeruginosa resistance against quinolones is closely evaluated and monitored. According to the ECDC 2014 surveillance data on antimicrobial resistance and antimicrobial consumption in Europe, the distribution of fluoroquinolone-resistant $P$. aeruginosa is decreasing in many European countries, the United States, and Canada, owing to the development of guidelines for appropriate antimicrobial use by the European Center for Disease Prevention and Control [4]. The fluoroquinolone resistance profile of $P$. aeruginosa strains present in companion dogs has also been evaluated in the United States, France, Brazil, Croatia, and Japan [5-9]. Resistance to fluoroquinolones differs from one country to another or depending on the year the study was performed. The fluoroquinolone resistance profile of $P$. aeruginosa in companion dogs has not been evaluated in South Korea, although enrofloxacin and marbofloxacin are commonly prescribed in veterinary medicine.

$P$. aeruginosa becomes resistant to quinolones through mutation of the quinolone resistance-determining region (QRDR) or plasmid-mediated quinolone resistance (PMQR). A QRDR mutation is a chromosomal point mutation of either DNA gyrase ( $g y r A$ and $g y r B$ ) or type IV topoisomerase (parC and parE), which is the main mechanism of resistance to quinolones in $P$. aeruginosa $[6,10]$. A previous study performed in the United States reported that half of the $102(51.0 \%)$ nalidixic acidresistant $P$. aeruginosa strains isolated from dogs had QRDR mutations [6]. QRDR mutations were also identified in fluoroquinolone-resistant $P$. aeruginosa from dogs in Brazil and Japan [7, 8]. However, the presence of QRDR mutations in $P$. aeruginosa in dogs has not been reported in South Korea. This study was performed to investigate the antimicrobial resistance and mutations in
gyrA and parC in fluoroquinolone-resistant $P$. aeruginosa from companion dogs in South Korea.

\section{Results \\ Distribution of $P$. aeruginosa strains isolated from companion dogs}

A total of 84 nonduplicated $P$. aeruginosa strains were isolated from 448 companion dogs either with or without clinical signs from 2017 to 2018. From 228 healthy dogs (healthy group), 38 strains $(16.7 \%)$ were detected in the ears (14 strains, $6.1 \%)$, eyes (10 strains, $4.4 \%)$, nasal cavity (7 strains, $3.1 \%$ ), or rectum (7 strains, $3.1 \%$ ). However, 46 strains $(17.7 \%)$ from 260 dogs with clinical signs (infected group) were isolated mostly from the ears (41 strains, $15.8 \%)$, and only a few were found in the genitalia (4 strains, $1.5 \%$ ) or pus (1 strain, $0.4 \%$ ) (Table 1 ). Among each specimen type collected from the infected group, $P$. aeruginosa were identified in $1.3 \%$ of isolates from genitalia (4/30), $31.5 \%$ of isolates from ears (41/ $130)$, and $1.3 \%$ of isolates from pus $(1 / 8)$. Therefore, $P$. aeruginosa was isolated mostly from ear samples (55 strains, $11.3 \%$ ) of companion dogs, which was independent of their health status.

\section{Antimicrobial resistance profile}

Strains were tested for resistance to 10 different antibiotics (Table 2, Fig. 1). In the healthy group, resistance to ciprofloxacin was the most frequent $(\mathrm{n}=4,10.5 \%)$, and resistance to ciprofloxacin-gentamicin-tobramycin, gentamicin, and tobramycin was observed for one organism. In the infected group, resistance to ciprofloxacin was the most frequent ( $n=7,15.2 \%$ ), followed by that to piperacillin $(n=3,6.5 \%)$, ciprofloxacin-gentamicin-tobramycin $(\mathrm{n}=2,4.3 \%)$, gentamicin $(\mathrm{n}=2,4.3 \%)$, tobramycin $(\mathrm{n}=2$, $4.3 \%)$ and amikacin $(\mathrm{n}=1,2.2 \%)$. No strain was resistant to cefepime, ceftazidime, colistin, imipenem, meropenem, or piperacillin-tazobactam. Additionally, resistance

Table 1 Distribution of nonduplicated Pseudomonas aeruginosa from healthy and infected dogs based on specimen types

\begin{tabular}{|c|c|c|c|}
\hline \multirow[t]{3}{*}{ Specimens } & \multicolumn{3}{|c|}{ No. (\%) isolates } \\
\hline & \multirow{2}{*}{$\begin{array}{l}\text { Healthy } \\
\text { group } \\
(n= \\
228)\end{array}$} & \multicolumn{2}{|l|}{ Infected group $^{a}$} \\
\hline & & Total $(n=260)$ & Specimen type \\
\hline Eye & $10(4.4)$ & $0(0.0)$ & $0 / 2(0.0)$ \\
\hline Rectum & $7(3.1)$ & $0(0.0)$ & 0/10 (0.0) \\
\hline Genitalia & - & $4(1.5)$ & $4 / 30(1.3)$ \\
\hline Ear & $14(6.1)$ & $41(15.8)$ & $41 / 130$ (31.5) \\
\hline Nasal cavity & $7(3.1)$ & $0(0.0)$ & 0/80 (0.0) \\
\hline Pus & - & $1(0.4)$ & 1/8 (1.3) \\
\hline Total & $38(16.7)$ & $46(17.7)$ & \\
\hline
\end{tabular}

${ }^{a}$ Disease name of the infected group: genitalia, pyometra; ear, otitis externa; pus, bronchitis 
Table 2 Antimicrobial resistance patterns of Pseudomonas aeruginosa from healthy and infected dogs

\begin{tabular}{llll}
\hline $\begin{array}{l}\text { Antimicrobial } \\
\text { resistance } \\
\text { pattern }^{a}\end{array}$ & \multicolumn{3}{l}{ No. (\%) resistant strains } \\
\cline { 2 - 4 } & $\begin{array}{l}\text { Healthy group } \\
(n=38)\end{array}$ & $\begin{array}{l}\text { Infected group } \\
(n=46)\end{array}$ & $\begin{array}{l}\text { Total } \\
(n=84)\end{array}$ \\
\hline PIP & $0(0.0)$ & $3(6.5)$ & $3(3.6)$ \\
CIP & $4(10.5)$ & $7(15.2)$ & $11(13.1)$ \\
CIP-GEN-TOB & $1(2.6)$ & $2(4.3)$ & $3(3.6)$ \\
AMK & $0(0.0)$ & $1(2.2)$ & $1(1.2)$ \\
GEN & $1(2.6)$ & $2(4.3)$ & $3(3.6)$ \\
TOB & $1(2.6)$ & $2(4.3)$ & $3(3.6)$ \\
\hline
\end{tabular}

${ }^{a} \mathrm{PIP}$, piperacillin (zone diameter resistance breakpoint, $\leq 14 \mathrm{~mm}$ ); GEN, gentamicin (zone diameter resistance breakpoint, $\leq 12 \mathrm{~mm}$ ); TOB, tobramycin (zone diameter resistance breakpoint, $\leq 12 \mathrm{~mm}$ ); AMK, amikacin (zone diameter resistance breakpoint, $\leq 14 \mathrm{~mm}$ ); CIP, ciprofloxacin (zone diameter resistance breakpoint, $\leq 15 \mathrm{~mm}$ )

to piperacillin and amikacin was observed only in strains from the infected group. While resistance to ciprofloxacin was apparently higher in the infected group than in the healthy group, there was no significant difference in the frequency of resistance to ciprofloxacin between the healthy and infected groups ( $p$-value 0.747$)$.

The MICs for 4 fluoroquinolone antibiotics, including nalidixic acid, were evaluated (Table 3). All $P$. aeruginosa strains from both groups were resistant to nalidixic acid (MIC ranges: 64 to $\geq 256 \mu \mathrm{g} / \mathrm{mL}$ in the healthy group and 32 to $\geq 256 \mu \mathrm{g} / \mathrm{mL}$ in the infected group). The highest percentage of resistance among the 4 fluoroquinolone agents was seen for enrofloxacin (breakpoint, $\geq 4 \mu \mathrm{g} / \mathrm{mL}$ ), with $15.8 \%$ (6/38) resistant strains from the healthy group and 37.0\% (17/46) from the infected group. The percentage of resistance to marbofloxacin $(\geq 4 \mu \mathrm{g} / \mathrm{mL}$ ) was $13.2 \%$ $(5 / 38)$ for strains from the healthy group and $26.1 \%$ $(12 / 46)$ for those from the infected group, and resistance $(\geq 8 \mu \mathrm{g} / \mathrm{mL})$ to levofloxacin was $13.2 \%(5 / 38)$ for the healthy group strains and $15.2 \%(7 / 46)$ for those from the infected group. Moreover, resistance $(\geq$ $4 \mu \mathrm{g} / \mathrm{mL}$ ) to ciprofloxacin was observed for $10.5 \%$ (4/ 38 ) of strains from the healthy group and $15.2 \%$ (7/ 46) of strains from the infected group (Table 3). Collectively, strains from the infected group were significantly more resistant to enrofloxacin and less susceptible to marbofloxacin than those from the healthy group $(p<0.05)$.

Amino acid variations in the gyrA and parC gene products Given that strains resistant to enrofloxacin among fluoroquinolone antibiotics were the most abundant, sequencing analysis of the gyrA and parC genes from the 23 enrofloxacin-resistant $P$. aeruginosa strains was performed (Table 4). QRDR mutations were identified in $6(15.8 \%)$ out of 38 strains from the healthy group and in 17 (37\%) out of 46 strains from the infected group; all 23 mutations belonged to the 23 enrofloxacin-resistant strains of $P$. aeruginosa. In addition, $11(47.8 \%)$ of these 23 strains were resistant to ciprofloxacin. Of the amino acid substitutions found in the 23 fluoroquinolone-resistant strains, 8 were present in the gyrA gene and 4 in the $\operatorname{parC}$ gene. Regarding gyrA, the mutation Thr83Ile was

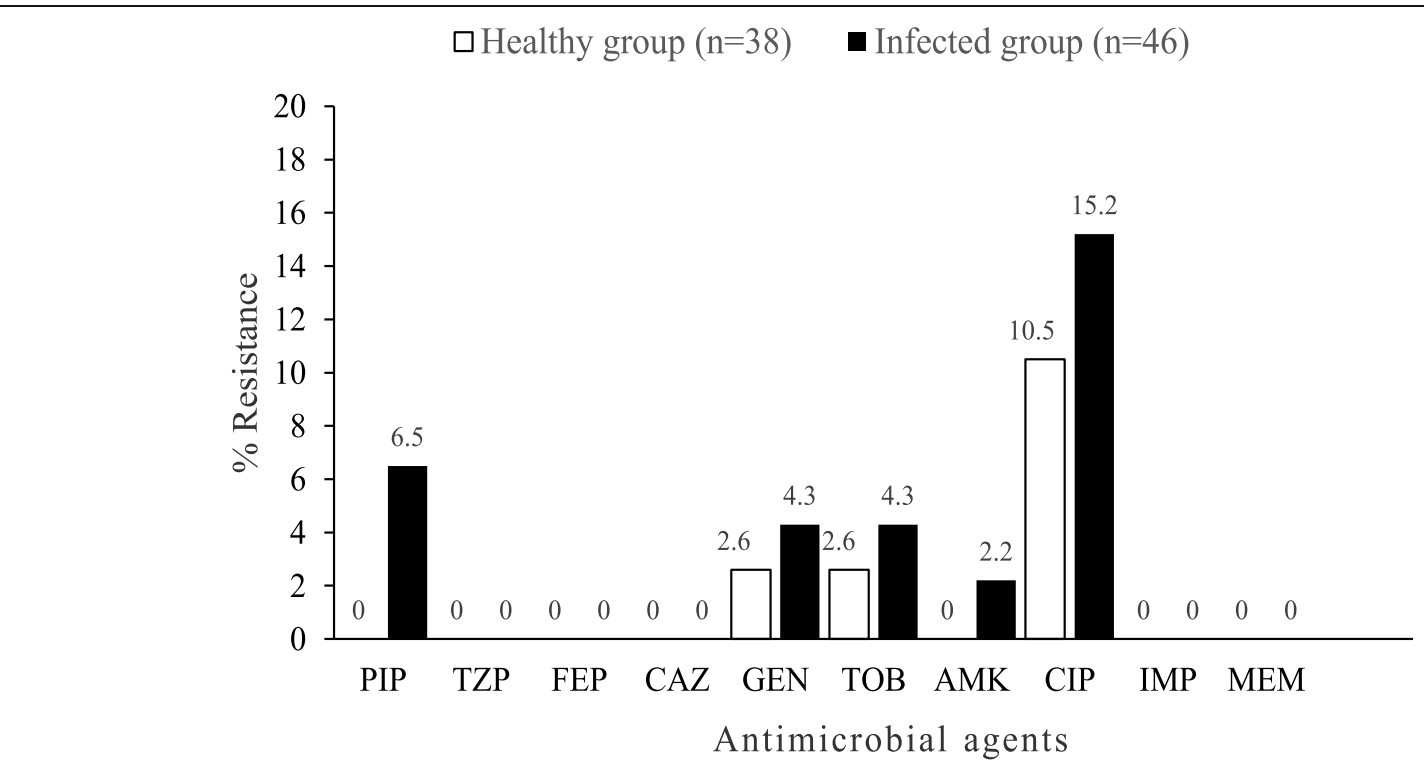

Fig. 1 Comparison of antimicrobial resistance of Pseudomonas aeruginosa from healthy and infected dogs. PIP, piperacillin; TZP, piperacillintazobactam; FEP, cefepime; CAZ, ceftazidime; GEN, gentamicin; TOB, tobramycin; AMK, amikacin; CIP, ciprofloxacin; IMP, imipenem; MEM, meropenem 
Table 3 Distribution of fluoroquinolone-resistant Pseudomonas aeruginosa from healthy and infected dogs

\begin{tabular}{|c|c|c|c|c|c|}
\hline \multirow{2}{*}{$\begin{array}{l}\text { Antimicrobial } \\
\text { agents }\end{array}$} & \multicolumn{2}{|c|}{$\mathrm{MIC}(\mu \mathrm{g} / \mathrm{mL})$} & \multicolumn{2}{|l|}{ No. (\%) resistant strains } & \multirow[t]{2}{*}{$p$-value } \\
\hline & Range & Breakpoint & Healthy group $(n=38)$ & Infected group $(n=46)$ & \\
\hline Nalidixic acid & $0.05-256$ & $\geq 32$ & $38(100.0)$ & $46(100.0)$ & $\mathrm{n} / \mathrm{a}$ \\
\hline Ciprofloxacin & $0.015-32$ & $\geq 4$ & $4(10.5)$ & $7(15.2)$ & 0.747 \\
\hline Levofloxacin & $0.015-32$ & $\geq 8$ & $5(13.2)$ & $7(15.2)$ & 0.700 \\
\hline Enrofloxacin & $0.03-16$ & $\geq 4$ & $6(15.8)$ & $17(35.4)$ & $0.031^{\circ}$ \\
\hline Marbofloxacin & $0.03-16$ & $\geq 4$ & $5(13.2)$ & $12(26.1)$ & 0.142 \\
\hline
\end{tabular}

a Statistically significant $(p<0.05)$

Table 4 Comparison of quinolone resistance-determining regions among fluoroquinolone-resistant Pseudomonas aeruginosa between healthy and infected dogs

\begin{tabular}{|c|c|c|c|c|c|c|}
\hline \multirow[t]{2}{*}{ Strain no. } & \multicolumn{2}{|l|}{ Amino acid substitution $^{a}$} & \multicolumn{4}{|c|}{$\operatorname{MIC}(\mu \mathrm{g} / \mathrm{mL})^{b}$} \\
\hline & gyrA & parC & $\mathrm{CIP}$ & LVX & ENR & MFX \\
\hline \multicolumn{7}{|l|}{ Healthy group } \\
\hline PAE18 & - & Pro116Arg & 4 & 16 & $\geq 16$ & $\geq 16$ \\
\hline PAE43 & Thr83lle & Pro116Arg & 2 & 8 & $\geq 16$ & 8 \\
\hline PAE51 & - & Pro116Arg & 16 & 16 & $\geq 16$ & $\geq 16$ \\
\hline PAE52 & Thr83lle & Ser87Leu, Pro116Arg & $\geq 32$ & $\geq 32$ & $\geq 16$ & $\geq 16$ \\
\hline PAE71 & - & Pro116Arg & $\geq 32$ & $\geq 32$ & $\geq 16$ & $\geq 16$ \\
\hline PAE85 & - & Pro116Arg & 0.25 & 0.5 & 4 & 1 \\
\hline \multicolumn{7}{|l|}{ Infected group } \\
\hline KVNON14 & Asp87Gly & Pro116Arg & 4 & 8 & $\geq 16$ & $\geq 16$ \\
\hline KVNON23 & Thr83lle & Pro116Arg & $\geq 32$ & $\geq 32$ & $\geq 16$ & $\geq 16$ \\
\hline KVNON33 & Thr83lle, Asp87Gly & Ser87Leu, Pro116Arg & $\geq 32$ & $\geq 32$ & $\geq 16$ & $\geq 16$ \\
\hline KVNON47 & - & Prol16fs & 0.5 & 2 & 4 & 2 \\
\hline KVNON66 & Asp87Gly & Pro116Arg & 4 & 8 & $\geq 16$ & $\geq 16$ \\
\hline KVNON127 & Asp87Tyr & Pro116Arg & 1 & 2 & 4 & 4 \\
\hline KVNON184 & - & Pro116Ala & 0.125 & 0.5 & 8 & 1 \\
\hline KVNON194 & Leu55Gln, Asp82Asn, Thr83Ala & Ser87Leu, Pro116Ala & $\geq 32$ & $\geq 32$ & $\geq 16$ & $\geq 16$ \\
\hline KVNON199 & Thr83lle & Pro116Arg & 8 & 32 & $\geq 16$ & $\geq 16$ \\
\hline KVNON216 & - & Pro116Arg & 0.5 & 2 & 4 & 2 \\
\hline KVNON219 & Thr83lle & Pro116Arg & 1 & 4 & 8 & 8 \\
\hline KVNON271 & - & Pro116Arg & 2 & 4 & 4 & 2 \\
\hline KVNON279 & Thr83lle & Pro116Arg & 4 & 8 & $\geq 16$ & $\geq 16$ \\
\hline KVNON324 & - & Pro116Arg & 0.5 & 4 & 4 & 4 \\
\hline KVNON442 & Asp87Asn & Pro116Arg & 0.5 & 2 & 4 & 4 \\
\hline KVNON508 & - & Pro116fs & 0.5 & 2 & 4 & 2 \\
\hline KVNON509 & - & Pro116Arg & 2 & 2 & 4 & 4 \\
\hline
\end{tabular}

${ }^{a}$ Amino acid substitutions in gyrA and parC: Thr83lle, ACC $\rightarrow$ ATC; Thr83Ala, ACC $\rightarrow$ GCC; Asp87Gly, GAC $\rightarrow$ GGC; Leu55Gln, CTG $\rightarrow$ CAG; Asp82Asn, GAC $\rightarrow$ AAC; Asp87Asn, GAC $\rightarrow$ AAC; Asp87Tyr, GAC $\rightarrow$ TAC; Pro116Arg, CCG $\rightarrow$ CGG; Pro116Ala, CCG $\rightarrow$ GCG; Ser87Leu, TGC $\rightarrow$ TTG; Pro116fs, CGG $\rightarrow$ CG-. Ala, alanine; Arg, arginine; Asn, asparagine; Asp, aspartic acid; Cys, cysteine; Gln, glutamine; Gly, glycine; lle, Isoleucine; Leu, leucine; Pro, proline; Ser, serine; Thr, threonine; Tyr, tyrosine; fs, frameshift

${ }^{b}$ NAL nalidixic acid, CIP ciprofloxacin, LVX levofloxacin, ENR enrofloxacin, MFX marbofloxacin 
found in 2 strains from the healthy group and in 4 strains from the infected group; in addition, other mutations were found in strains from the infected group: Asp87Gly in 2 strains, Thr83Ile-Asp87Gly in 1 strain, Leu55Gln-Asp82Asn-Thr83Ala in 1 strain, and Asp87Asn in 1 strain. Notably, the novel triplenucleotide mutation found in gyrA, leading to codon changes Leu $55 \rightarrow$ Gln, Asp $82 \rightarrow$ Asn, and Thr83 $\rightarrow$ Ala, corresponds to a $P$. aeruginosa strain from the infected group with high MIC values for the four fluoroquinolone agents tested. Moreover, the strain with two nucleotide mutations, i.e., Thr83Ile and Asp87Gly, showed identical MIC values for these four antibiotics. Mutations in gyrA were significantly more common in strains from the infected group than in those from the healthy group $(p<0.05)$.

Regarding the $\operatorname{parC}$ gene, mutations were observed in all 23 enrofloxacin-resistant $P$. aeruginosa strains. A novel single nucleotide mutation at parC codon 116, resulting in a Pro116 $\rightarrow$ Arg mutation, was found in 21 (91.3\%) of the strains, which included a double nucleotide alteration at codons 87 and 116, Ser87 $\rightarrow$ Leu and Pro116 $\rightarrow$ Arg, that was observed in 1 strain from the healthy group and in 2 strains from the infected group. In addition, a frameshift ( $\mathrm{fs}$ ) mutation due to a nucleotide deletion at parC codon 116, Pro116fs, CGG $\rightarrow$ CG-, was detected in 2 strains from the infected group. Therefore, mutation hotspots found for $\operatorname{gyr} A$ and $\operatorname{parC}$ were Thr83Ile $(n=7)$ and Pro116Arg $(n=21)$, respectively. There was no significant difference between the number of point mutations in either the $g y r A$ or the $\operatorname{parC}$ genes and the MIC values for fluoroquinolone antimicrobial agents among resistant strains. In addition, QRDR mutations in the gyrA or parC genes conferring intermediate resistance to enrofloxacin (MIC: 1 to $2 \mu \mathrm{g}$ / $\mathrm{mL}$ ) were not found in the $P$. aeruginosa strains.

\section{Discussion}

$P$. aeruginosa often causes otitis externa/media and cystitis in companion dogs. Despite the regular usage of fluoroquinolone agents, such as enrofloxacin and marbofloxacin, in the treatment of infected dogs, neither the antimicrobial susceptibility nor the quinolone-resistance profile of $P$. aeruginosa strains has ever been studied in South Korea. This study aimed to reveal the antimicrobial susceptibility and quinolone resistance of $P$. aeruginosa strains isolated from both healthy and clinically ill companion dogs. P. aeruginosa was recovered from several samples in dogs in the healthy group; nevertheless, one-third of these strains were obtained from ears. In addition, $P$. aeruginosa was isolated mainly from the ears of dogs in the infected group. These results are consistent with $P$. aeruginosa as a common agent causing otitis. The fact that one-third of the strains from the healthy group were obtained from ears suggests a history of otitis externa/media in dogs that appear to be healthy.

$P$. aeruginosa strains from this study were not resistant to a variety of antimicrobials, as tested by disk diffusion assays, except for ciprofloxacin, gentamicin, tobramycin, and amikacin. The resistance to these latter antimicrobial agents may be due to the prevalence of antimicrobial use for treating diseases in animal hospitals. It is important to note that a significant difference in the resistance frequencies to ciprofloxacin between the healthy and infected groups was not found. These results could be explained if the usage of ciprofloxacin, among quinolone antibiotics, is less common in animal hospitals for treating diseases; then, it would be possible to hypothesize that the companion dogs in this study had not been exposed to ciprofloxacin.

MIC values for fluoroquinolones were different among antibiotics. All strains showed high resistance rate to enrofloxacin but low resistance rate to ciprofloxacin. These results are similar to $P$. aeruginosa resistance trends reported in other countries. Previous resistance reports indicate that $13.1 \%$ of strains from this study, $16 \%$ from the United States, 13\% from Spain, 2.2\% from Croatia, 4.8\% from Brazil, and 20\% from Japan are resistant to ciprofloxacin [6-9]. Moreover, the reported percentages of resistance to enrofloxacin and marbofloxacin were 27.4 and $20.2 \%$ in this study, 31 and $27 \%$ in the United States, 21.7 and 9\% in Spain, and 15.6 and 8.9\% in Brazil, respectively, whereas $31.5 \%$ resistance to enrofloxacin was reported in Japan. According to a previous study, $P$. aeruginosa strains show their highest resistance to enrofloxacin, the most commonly used fluoroquinolone in veterinary medicine [6]. In Croatia, resistance to enrofloxacin and marbofloxacin significantly increased from 2.7 to $15.6 \%$ and from 4.4 to $8.9 \%$, respectively, since the use of marbofloxacin, in addition to enrofloxacin, in veterinary medicine was approved in the country $[9,11]$. Our results show that resistance to marbofloxacin was $20.2 \%$, which is higher than that reported for strains from Spain and Croatia [8,9]. This finding may be related to differences in the frequency of marbofloxacin prescription in each country. Since the usage of marbofloxacin for treating clinically ill dogs was approved in South Korea, follow-up studies should be undertaken to determine the evolution of fluoroquinolone-resistant $P$. aeruginosa strains.

Previous studies have also reported different susceptibilities or resistances to enrofloxacin depending on the origin of the studied isolates; ear isolates, for example, show a significantly higher resistance to enrofloxacin than skin isolates [12]. Other studies have reported increased resistance rates in ear isolates $[6,13]$. In Croatia, resistance to enrofloxacin increased from $1.0 \%$ in 2011 to $8.9 \%$ in 2017, perhaps because Mekić et al. evaluated 
only the resistance of isolates from dogs with otitis externa $[9,14] . P$. aeruginosa strains isolated from ears in this study showed a high susceptibility to ciprofloxacin $(82.5 \%)$ and a low susceptibility to both enrofloxacin (17.5\%) and marbofloxacin (3.5\%). This trend is similar to that reported in Croatia, where the use of marbofloxacin has been licensed only for treating otitis externa. As mentioned, in this study, strains from ears were significantly less susceptible to marbofloxacin than isolates from other body parts $(p<0.05)$. This finding suggests that caution should be taken in the usage of enrofloxacin or marbofloxacin in dogs with otitis to avoid an increase in rates of resistance to these antibiotics.

In the present study, novel QRDR mutations in both the gyrA and parC genes were detected in fluoroquinolone-resistant $P$. aeruginosa. Among QRDR genes (gyrA, gyrB, parC, and parE), QRDR mutations are most frequently detected in $g y r A$ and parC [6-8]. In this study, the same point mutation within parC was found in all enrofloxacin-resistant strains, whereas point mutations in $g y r A$ were almost exclusively detected in strains from the infected group. The most commonly reported point mutations or hotspots are Thr83 and Asp87 for gyrA and Ser87 for parC in both dogs and humans $[6,8,15]$. Consistently, the hotspot for gyrA was Thr83 $(n=8,38.1 \%)$ in this study. In contrast, a point mutation at Pro116 of $\operatorname{parC}(n=21,80.8 \%)$ in this study was the most frequently observed mutation found in strains from this study. This sequence may be a wild-type allele of canine $P$. aeruginosa strains from South Korea rather than a QRDR mutation resulting in increased resistance to fluoroquinolones. Nevertheless, a high correlation between QRDR mutations and increased MICs to fluoroquinolones has been reported previously [7]. In this study, the MIC values for the four fluoroquinolone antibiotics studied were not significantly different for strains with different gyrA mutations, while parC mutations were detected in all 23 enrofloxacin-resistant strains. As a result, the novel Pro116 alteration described in $\operatorname{parC}$, which has never been reported in nalidixic acid-resistant gram-negative bacteria until now, is suspected to be correlated with increased enrofloxacin resistance.

\section{Conclusions}

The high resistance to enrofloxacin and the occurrence of QRDR mutations in P. aeruginosa strains from clinically ill dogs may reflect the increasing use of enrofloxacin in animal hospitals. However, further investigation is warranted regarding the pattern and frequency of antimicrobial use in dogs. Overall, this study provides a warning about the inappropriate use of fluoroquinolone agents in the treatment of pseudomonal infections in companion dogs.

\section{Methods \\ Sample collection}

To investigate the antimicrobial susceptibility and fluoroquinolone resistance mechanisms of $P$. aeruginosa strains isolated from companion dogs with or without present clinical signs, sampling was performed on a total of 50 veterinary hospitals from five Korean provinces between 2017 and 2018. Samples were collected from the ears, nasal cavity, eyes, and rectum of dogs that were clinically healthy and had not taken any antibiotics within the last six months, using sterile transport swabs (COPAN, Brescia, Italy). Using the same sampling method, samples from the ears, nasal cavity, eyes, genitalia, rectum, and pus were collected from dogs with clinical signs of bacterial infection. Isolates were first classified according to their origin as belonging either to the healthy group or to the infected group. All samples collected from companion animals were collected under the owner's approval. Every individual was provided with information regarding the purpose and method of sampling. The need for ethics approval was deemed unnecessary according to the national regulations.

\section{Isolation and identification}

Swab samples were cultured on trypticase soy broth (TSB, Becton, Dickinson and Company, Sparks, MD, USA) for $18 \mathrm{~h}$ at $37^{\circ} \mathrm{C}$. Then, they were streaked on MacConkey agar (Becton, Dickinson and Company) and incubated for $18 \mathrm{~h}$ at $37^{\circ} \mathrm{C}$. Those isolates that were identified as $P$. aeruginosa by Gram staining (Gram stain set, Remel, Dartfold, UK), oxidase tests (Becton, Dickinson and Company), and a microbial identification system (VITEK $^{\circ}$ MS, bioMérieux, Marcy-l'Étoile, France) were selected for further analysis.

\section{Determination of antimicrobial susceptibility and minimum inhibitory concentrations}

Antimicrobial susceptibility analyses were performed by the disk diffusion test, according to the Clinical Laboratory of Standard Institute guidelines [16]. Eleven different antimicrobial disks were used (Oxoid Limited, Basingstoke, UK) for testing: piperacillin, piperacillintazobactam, cefepime, ceftazidime, ciprofloxacin, gentamicin, amikacin, tobramycin, meropenem, and imipenem.

The minimum inhibitory concentrations (MICs) for fluoroquinolone antimicrobials were determined as indicated by the CLSI broth microdilution method. The quinolones and fluoroquinolones used were nalidixic acid (Sigma-Aldrich, Germany), ciprofloxacin (Sigma-Aldrich), levofloxacin (Sigma-Aldrich), enrofloxacin (Bayer 
Vital GmbH, Leverkusen, Germany), and marbofloxacin (Vetoquinol, Lure, France). P. aeruginosa ATCC 27853 and Escherichia coli ATCC 25922 were used as quality control strains. The breakpoints for ciprofloxacin $(\geq 4 \mu \mathrm{g} /$ $\mathrm{mL})$ and levofloxacin $(\geq 8 \mu \mathrm{g} / \mathrm{mL})$ were followed based on CLSI guidelines, whereas the breakpoints for nalidixic acid $(\geq 32 \mu \mathrm{g} / \mathrm{mL})$ was followed as described by Rubin et al., 2008 and for enrofloxacin $(\geq 4 \mu \mathrm{g} / \mathrm{mL})$ and marbofloxacin $(\geq 4 \mu \mathrm{g} / \mathrm{mL})$ as described by Pintarić et al., 2017.

\section{PCR amplification and sequencing analysis}

Genomic DNA was extracted from all $P$. aeruginosa strains using a QIAamp DNA Mini kit (QIAGEN, Hilden, Germany) according to the manufacturer's instructions. PCR amplification of the $\operatorname{gyr} A$ and $\operatorname{parC}$ genes was performed as previously described (Rubin et al., 2008). Purified PCR products were sequenced using an automated 3730xl DNA Analyzer (Applied Biosystems, Foster City, CA, USA), and the resulting sequences were compared against the complete genome sequence of $P$. aeruginosa ATCC 27853 (GenBank accession number, CP015117) using both the BLAST network service and the ClustalW multiple sequence alignment program (www.genome.jp/toolsbin/clustalw).

\section{Statistical analysis}

The obtained data are presented as the relative frequency from the values. A $t$-test and Pearson's Chisquare test were used to compare the antimicrobial susceptibility profiles between the healthy group and the infected group. All statistical analyses were performed by using SPSS 20.0 software (IBM SPSS, Inc., Chicago, IL, USA). The results were considered statistically significant for $p$ values less than 0.05 .

\section{Nucleotide sequence accession numbers}

Newly described sequences for the gyrA and parC genes have been assigned the following GenBank accession numbers: MN068218 to MN068220 (parC mutations) and MN068221 (gyrA mutation).

\footnotetext{
Abbreviations

Ala: Alanine; AMK: Amikacin; Arg: Arginine; Asn: Asparagine; Asp: Aspartic acid; CIP: Ciprofloxacin; CLSI: Clinical and Laboratory Standards Institute; Cys: Cysteine; ECDC: European Centre for Disease Prevention and Control; ENR: Enrofloxacin; Fs: Frameshift; GEN: Gentamicin; GIn: Glutamine; Gly: Glycine; lle: Isoleucine; Leu: Leucine; LVX: Levofloxacin; MFX: Marbofloxacin; MIC: Minimum inhibitory concentration; NAL: Nalidixic acid; PCR: Polymerase chain reaction; PIP: Piperacillin; Pro: Proline; QRDR: Quinolone resistance-determining region; Ser: Serine; Thr: Threonine; TOB: Tobramycin; Tyr: Tyrosine
}

\section{Availability of data and material}

The datasets generated and/or analyzed during the current study are available in the GeneBank repository [Accession no. MN068218, MN068219, MN068220, MN068221] and from the corresponding author on reasonable request.

\section{Authors' contributions}

YP performed the antibiotic susceptibility testing and identification of QRDR mutations, analyzed all relevant data and mainly wrote this manuscript. JO and HP guided and reviewed the design of this study and edited the manuscript. SP, SS, WS, and JC contributed to the isolation, identification, and antimicrobial susceptibility testing (disk diffusion test only) of bacterial strains. All authors read and approved the final manuscript.

\section{Funding}

The Research of Korea Centers for Disease Control and Prevention supported this manuscript by funding (2017 N-ER5405-02) the costs for experimental equipment, promoting collaboration between veterinary and human medicine in study design and sample collection as part of 'Study on epidemiological relationships among major antimicrobial resistant bacteria from companion animals, environments, and people in veterinary hospitals'.

\section{Ethics approval and consent to participate}

All samples collected from companion animals were collected under the owner's approval in written consent. Every individual was provided with a document explaining the purpose and method of sample collection. Furthermore, ethics approval was waivered by Institutional Review Board under [Exempt review categories of research] (Korean Council of Science Editors, https://www.kcse.org/books/SCBK1000146).

\section{Consent for publication}

Not applicable.

\section{Competing interests}

None of the authors have any other financial or personal relationships that could inappropriately influence or bias the content of the paper.

\section{Author details}

${ }^{1}$ Department of Veterinary Internal Medicine, Konkuk University College of Veterinary Medicine, Seoul, South Korea. ${ }^{2}$ Department of Laboratory Medicine, Hallym University College of Medicine, Chuncheon, South Korea.

${ }^{3}$ Biotechnology Division, Jeonbuk National University, Iksan, South Korea.

Received: 11 August 2019 Accepted: 25 March 2020

Published online: 15 April 2020

\section{References}

1. Kümmerer K. Resistance in the environment. J Antimicrob Chemother. 2004; 54:311-20.

2. Hammerum AM, Heuer OE. Human health hazards from antimicrobialresistant Escherichia coli of animal origin. Clin Infect Dis. 2009;48:916-21.

3. Costa PM, Loureiro L, Matos AJ. Transfer of multidrug resistant bacteria between intermingled ecological niches: the interface between humans, animals and the environment. Int J Environ Res Public Health. 2013;10:27894.

4. European Centre for Disease Prevention and Control. Antimicrobial resistance surveillance in Europe 2014. Annual Report of the European Antimicrobial Resistance Surveillance Network (EARS-Net). Stockholm: ECDC; 2015

5. Meunier D, Acar JF, Martel JL, Kroemer S, Valle M. 2004. A seven-year survey of susceptibility to marbofloxacin of pathogenic strains isolated from pets. Int J Antimicrob Agents. 2004;24:592-8.

6. Rubin J, Walker RD, Blickenstaff K, Bodeis-Jones S, Zhao S. Antimicrobial resistance and genetic characterization of fluoroquinolone resistance of Pseudomonas aeruginosa isolated from canine infections. Vet Microbiol. 2008;131:164-72.

7. Harada K, Arima S, Nina A, Kataoka Y, Takahashi T. Characterization of Pseudomonas aeruginosa isolates from dogs and cats in Japan: current status of antimicrobial resistance and prevailing resistance mechanisms. Microbiol Immunol. 2012;56:123-7. 
8. Arais LR, Barbosa AV, Carvalho CA, Cerqueira AM. Antimicrobial resistance, integron carriage, and gyrA and gyrB mutations in Pseudomonas aeruginosa isolated from dogs with otitis externa and pyoderma in Brazil. Vet Dermatol. 2016:27:113-7e31.

9. Pintarić S, Matanović K, Martinec BS. Fluoroquinolone susceptibility in Pseudomonas aeruginosa isolates from dogs-comparing disk diffusion and microdilution methods. Veterinarski Arhiv. 2017:87:291-300.

10. Jiang $X, Y u T$, Jiang $X$, Zhang W, Zhan L, Ma J. Emergence of plasmidmediated quinolone resistance genes in clinical isolates of Acinetobacter baumannii and Pseudomonas aeruginosa in Henan. China Diagn Microbiol Infect Dis. 2014;79:381-3.

11. Seol B, Naglić T, Madić J, Bedeković M. In vitro antimicrobial susceptibility of 183 Pseudomonas aeruginos a strains isolated from dogs to selected antipseudomonal agents. J Vet Med B Infect Dis Vet Public Health. 2002;49: 188-92.

12. Wildermuth BE, Griffin CE, Rosenkrantz WS, Boord MJ. Susceptibility of Pseudomonasisolates from the ears and skin of dogs to enrofloxacin, marbofloxacin, and ciprofloxacin. J Am Anim Hosp Assoc. 2007;43:337-41.

13. McKay L, Rose CD, Matousek JL, Schmeitzel LS, Gibson NM, Gaskin JM. Antimicrobial testing of selected fluoroquinolones against Pseudomonas aeruginosa isolated from canine otitis. J Am Animal Hosp Assoc. 2007;43: 307-12.

14. Mekić S, Matanović K, Šeol B. Antimicrobial susceptibility of Pseudomonas aeruginosa isolates from dogs with otitis externa. Vet Rec. 2011;169:125.

15. Farahi RM, Ali AA, Gharavi S. Characterization of gyrA and parC mutations in ciprofloxacin-resistant Pseudomonas aeruginosa isolates from Tehran hospitals in Iran. Iran J Microbiol. 2018;10:242-9.

16. CLSI. Performance Standards for Antimicrobial Susceptibility Testing. 27th ed. CLSI supplement M100. Wayne: Clinical and Laboratory Standards Institute; 2017. .

\section{Publisher's Note}

Springer Nature remains neutral with regard to jurisdictional claims in published maps and institutional affiliations.

Ready to submit your research? Choose BMC and benefit from:

- fast, convenient online submission

- thorough peer review by experienced researchers in your field

- rapid publication on acceptance

- support for research data, including large and complex data types

- gold Open Access which fosters wider collaboration and increased citations

- maximum visibility for your research: over $100 \mathrm{M}$ website views per year

At $\mathrm{BMC}$, research is always in progress.

Learn more biomedcentral.com/submissions 